\title{
A Novel Gene Mutation of PIK3R1EY451delinsD in Breast Cancer with the Resistance to HER2-targeted Therapy
}

\author{
Kainan Wang ${ }^{1}$, Xuelu $\mathrm{Li}^{1}$, and Man $\mathrm{Li}^{2}$ \\ ${ }^{1}$ The Second Hospital of Dalian Medical University \\ ${ }^{2}$ Affiliation not available
}

October 16, 2020

\begin{abstract}
Previously, little is known about the relationship between the PIK3R1 mutation status and the resistance to HER2-targeted therapies. Herein, we report a HER2-positive breast cancer patient with PIK3R1EY451delinsD showed resistance to HER2targeted therapy, and adding everolimus to the treatment of trastuzumab and carboplatin achieved better effect.
\end{abstract}

\section{Introduction}

HER2-positive breast cancer occurs in approximately $20 \%$ of all breast cancers, and HER2-targeted therapies, such as trastuzumab and lapatinib, have shown encouraging efficacy in HER2-positive breast cancer among the adjuvant and advanced disease settings ${ }^{1}$. However, more than $50 \%$ HER2-positive breast cancer patients are inevitable to generate resistance to the anti-HER2 therapy ultimately ${ }^{1}$. The PI3K/AKT pathway is one of the main downstream signaling pathways of HER2, and therefore the activation of the PI3K signaling pathway associates with resistance to HER2-targeted treatments ${ }^{2,3}$. There is less research that reported the correlation between PIK3R1 mutation status and the resistance to HER2-targeted therapy in breast cancer $^{4}$. PIK3R1 mutations are only found in a small subset $(1.8 \%, 87 / 4602$, TCGA_all breast cancer studies) of breast cancers ${ }^{5}$. Intriguingly, PIK3R1 mutations were more prevalent (17\%, 25/147, FUSCC) in the Chinese cohort ${ }^{6}$. Here we describe a HER2-positive breast cancer patient harboring PIK3R1 ${ }^{\text {EY }}$ 451delinsD who developed resistance to HER2-targeted therapy. This provides a novel clue that PIK3R1EY451delinsD mutation might be clinically useful to choose the treatment option of everolimus for HER2 positive breast cancer.

We present the following case in accordance with the CARE reporting checklist.

\section{Case report}

We showed that a 52-year-old HER2-positive breast cancer patient underwent left breast modified radical mastectomy, and was diagnosed in December 2014 with left pT1N2M0, which immunological histological chemistry (IHC) confirmed ER-negative, PR-negative, HER2-positive 3+ (FISH+), Ki67 50\%. She was then treated with 8-cycles of doxorubicin/cyclophosphamide followed by paclitaxel plus trastuzumab (ACTH) and radiotherapy (DT) (Figure 1A). However, the PET-CT scan revealed lung metastases in January 2015. Subsequently, the patient received 4-cycles of gemcitabine/capecitabine /trastuzumab $(\mathrm{G}+\mathrm{X}+\mathrm{H})$ and approximately ten months of lapatinib/capecitabine (L+Cap) (Figure 1A), but both options achieved progressive disease (PD) as assessed by computed tomography (CT) scans using the RECIST criteria (1.1) 7. In December 2016, metastases appeared in many organs of the body, including liver, lung and brain. To ease the neurological symptoms, the patient received the whole brain radiation therapy (WBRT) and 2cycles of trastuzumab/navelbine (H+NVB) for metastatic disease. Disappointingly, the patient continuously progressed (Figure 1A). 
Looking forward to identify other therapeutic options, the archived primary breast tumor tissue and the peripheral blood samples were collected for 425 cancer-related genes sequencing (Geneseeq, Nanjing, China) (Figure 1B ), which showed ERBB2 magnification consistent with the result of HER2-positive FISH. Intriguingly, the PIK3R1 p.EY451delinsD (c.1352_1354delAAT) and TP53 p.V173M (c.G517A) mutations were also detected at the high allele frequency (Figure 1B ). Due to the identification of the PIK3R1 mutation and considering the increased PI3K pathway activity, we thought that the combination treatment of trastuzumab and everolimus (mTOR inhibitor) was worth applying. Therefore, the patient began the fourth-line treatment, everolimus was given combining with trastuzumab and carboplatin $(\mathrm{H}+\mathrm{EVER}+\mathrm{CBP})$ for eight cycles (Figure 1A). After eight cycles of everolimus containing regimen, CT and MRI scans revealed the decreased lung metastasis and the stable liver lesions (Figure 1A). A peripheral blood sample collected again after eight cycles was profiled using the Geneseeq ctDNA assay, which revealed the same PIK3R1 p.EY451delinsD mutation along with the persistent brain metastasis growth (Figure 1). Unfortunately, the $\mathrm{H}+\mathrm{EVER}+\mathrm{CBP}$ therapy could not block the intracranial tumor growth and the patient died shortly thereafter.

\section{Discussion}

Trastuzumab and Lapatinib have been the mainstays of anti-HER2 treatment in breast cancer. While they showed superior efficacy in subsets of primary and metastatic HER2-positive breast cancers, therapeutic resistance to HER2-targeted therapy remains an important clinical problem. Expression of mutant PIK3R1 in endometrial cancer ${ }^{8,9}$, malignant glioma ${ }^{10}$ and breast cancer cell lines ${ }^{6,11,12}$ is associated with activation of PI3K downstream signaling. However, the clinical significance of PIK3R1 mutations in HER2-positive breast cancer patients is less well understood and needs further investigation. For the first time, we reported a case of a 52-year-old HER2-positive breast cancer patient with a novel PIK3R1 ${ }^{\text {EY451delinsD }}$ who developed resistance to HER2-targeted therapies. Strikingly, the combined use of trastuzumab and everolimus overcame the resistance in this case harboring PIK3R1 ${ }^{\text {EY451delinsD }}$.

The precise knowledge of the genomic alterations present in cancer is critical to select the optimal treatment for each patient, so monitoring the impact of individual oncogenic alterations on lesion specific responses is indispensable. Cheung et al. and Urick et al. reported that thePIK3R1 indels (E439del, H450-E451del, Y463-L466del, R574fs, T576del, R574-T576del) have shown to be oncogenic and led to increased levels of p-AKT in endometrial cancer ${ }^{8,9}$. Quayle et al. demonstrated that three PIK3R1 indels (DKRMNS560del, R574fs, T576del) with the strongest activities increased P110 $\alpha$ kinase activity in the in vitro kinase assay in malignant glioma ${ }^{10}$. Cheung et al. uncovered that the PIK3R1 R348* and L370fs truncations were localized to the nucleus, where they facilitated abnormal pathway activation ${ }^{11}$. Thorpe et al. and their colleagues found that the genetic ablation of PIK3R1 accelerated a mouse model of HER2/neu-driven breast cancer ${ }^{12}$. Chen et al. ${ }^{6}$ provided a landscape of PIK3R1 mutations in 149 Chinese breast cancer patients, and reported that PIK3R1 mutations were prevalent (25 tumors, $17 \%$ ) in this Chinese cohort. They also confirmed when normal mammary epithelial cell line MCF10A overexpressing exogenous PIK3R1 mutations were grown in the absence of EGF, the levels of pAKT ${ }^{\text {Ser473 }}$ were slightly higher ${ }^{6}$. Together, there is an increasing number of evidence that PIK3R1 mutations activate the PI3K signaling pathway and display oncogenic properties $3,4,6,8-13$.

By analyzing the cBioPortal database, there is a huge difference of frequency range of PIK3R 1 alterations among types of cancer from more than $30 \%$ to less than $2 \%$ (Figure $2 \mathrm{~A}$ ). It is also worth noting that there are about 31\% PIK3R1 mutations (Uterine_TCGA PanCan, Figure 2A) that frequently occur in uterine corpus endometrial carcinoma. Future PIK3R1 mutational location analysis that covers all mutations is shown as Figure 2B, including 65939 cancer patients and 1690 mutations. In addition, we also summary characteristics of eleven patients with the same E541_Y542del from cBioPortal (Figure 2C), but PIK3R1 ${ }^{\text {EY451delinsD is a }}$ rare mutant form with little prior knowledge in breast cancer patients. To better display the result of PIK3R1 mutations, we showed that genomic characterization of PIK3R1 in breast cancer cohorts (Figure $2 \mathrm{D}$ and $2 \mathrm{E})$. PIK3R1 mutations are only found in a small subset $(1.8 \%, 87 / 4602$, www.cbioportal.org) of breast cancers including HER2 positive subtype (Figure 2E). In contrast, PIK3R1 mutations (not including 
PIK3R1 $1^{\text {EY451delinsD }}$ ) are prevalent (25 tumors, $17 \%$ ) in Chinese breast cancer patients ${ }^{6}$. This is the first report representing PIK3R1 ${ }^{\text {EY451delinsD }}$ in breast cancer.

This novel PIK3R1 ${ }^{\text {EY451delinsD }}$ confers resistance to trastuzumab and lapatinib in HER2-positive breast cancer. Fortunately, this case supports the combined use of trastuzumab and everolimus after failure of previous targeted therapies in HER2-positive breast cancer patient harboring PIK3R $1^{\text {EY 451delinsD }}$. However, brain metastasis was very tricky to treat in clinical, and we needed more effective drugs to cross the bloodbrain barrier. We believe that PIK3R1 alterations can be clinically useful to predict the treatment response for HER2-targeted treatment. In the future, functional PIK3R1 alterations should be incorporated in clinical trials to evaluate treatment response of targeted therapies in HER2-positive breast cancer.

Preclinical studies have demonstrated that breast cancer cell lines harboring PIK3R1 mutants or loss of p85 $\alpha$ (encoded by PIK3R1 gene) activate the PI3K/AKT signaling pathway ${ }^{6,12}$. However, whether PIK3R1 mutation status confers resistance to HER2-targeted therapies still awaits testing in HER2 positive breast cancer patients. Our case validated PIK3R1 $1^{\text {EY451delinsD }}$ as a potential mechanism of resistance to HER2targeted inhibition, and suggested that PIK3R1 alterations may also be clinically useful to stratify the treatment option for HER2-targeted therapies or in combination with everolimus treatment.

Abbreviations

ctDNA: circulating tumor DNA

$\mathrm{AF}$ : allele frequency.

AC: doxorubicin/cyclophosphamide

TH: paclitaxel/trastuzumab

DT: radiotherapy

$\mathrm{G}+\mathrm{X}+\mathrm{H}$ : gemcitabine/xeloda/trastuzumab

L+Cap: Lapatinib/capecitabine

WBRT: whole brain radiation therapy

NVB: navelbine

EVE: everolimus

CBP: Carboplatin

FUSCC: Fudan University Shanghai Cancer Center

PI3K: phosphatidylinositol-3-kinase

\section{Acknowledgments}

We would like to thank Qiwei Pan for their expert technical assistance and helpful comments.

\section{Author contribution}

X. L. and K.W. participated in clinical therapy and statistical analysis. X.L., K.W. and M.L. wrote the manuscript. M.L. and Z.Z. revised the manuscript. All authors read and approved the final manuscript.

\section{Conflict of Interest}

The authors declare no conflict of interest.

\section{Disclosure}

Funding:This work was supported by the National Natural Science Foundation of China (NO.81872756 to Man Li). 
Ethical Statement: All of the intended procedures in the present study, including the use of specimens from human subjects, were approved with the written informed consent of the patient. The experimental protocol was approved by the Dalian Medical University Ethics Committee.

\section{References}

1. Tebbutt N, Pedersen MW and Johns TG. Targeting the ERBB family in cancer: couples therapy. Nature reviews Cancer. 2013; 13: 663-73.

2. Thorpe LM, Yuzugullu $\mathrm{H}$ and Zhao JJ. PI3K in cancer: divergent roles of isoforms, modes of activation and therapeutic targeting. Nature reviews Cancer . 2015; 15: 7-24.

3. Dornan GL and Burke JE. Molecular Mechanisms of Human Disease Mediated by Oncogenic and Primary Immunodeficiency Mutations in Class IA Phosphoinositide 3-Kinases. Frontiers in immunology . 2018; 9: 575 .

4. Cheung LW and Mills GB. Targeting therapeutic liabilities engendered by PIK3R1 mutations for cancer treatment. Pharmacogenomics . 2016; 17: 297-307.

5. Comprehensive molecular portraits of human breast tumours. Nature . 2012; 490: 61-70.

6. Chen L, Yang L, Yao L, et al. Characterization of PIK3CA and PIK3R1 somatic mutations in Chinese breast cancer patients. Nat Commun . 2018; 9: 1357.

7. Nishino M, Jackman DM, Hatabu H, et al. New Response Evaluation Criteria in Solid Tumors (RECIST) guidelines for advanced non-small cell lung cancer: comparison with original RECIST and impact on assessment of tumor response to targeted therapy. AJR American journal of roentgenology . 2010; 195: W221-8.

8. Cheung LW, Hennessy BT, Li J, et al. High frequency of PIK3R1 and PIK3R2 mutations in endometrial cancer elucidates a novel mechanism for regulation of PTEN protein stability. Cancer Discov . 2011; 1: $170-85$.

9. Urick ME, Rudd ML, Godwin AK, Sgroi D, Merino M and Bell DW. PIK3R1 (p85alpha) is somatically mutated at high frequency in primary endometrial cancer. Cancer Res . 2011; 71: 4061-7.

10. Quayle SN, Lee JY, Cheung LW, et al. Somatic mutations of PIK3R1 promote gliomagenesis. PloS one . 2012; 7: e49466.

11. Cheung LW, Yu S, Zhang D, et al. Naturally occurring neomorphic PIK3R1 mutations activate the MAPK pathway, dictating therapeutic response to MAPK pathway inhibitors. Cancer cell . 2014; 26: 479-94.

12. Thorpe LM, Spangle JM, Ohlson CE, et al. PI3K-p110alpha mediates the oncogenic activity induced by loss of the novel tumor suppressor PI3K-p85alpha. Proc Natl Acad Sci U S A . 2017; 114: 7095-100.

\section{Figure Legends}

\section{Figure 1. Basic Information of the patient.}

(A) The treatment timelines, and images of brain, liver and lung metastases. AC, doxorubicin/cyclophosphamide; TH, paclitaxel/trastuzumab; DT, radiotherapy; $\mathrm{G}+\mathrm{X}+\mathrm{H}, \quad$ gemcitabine/xeloda/trastuzumab; L+Cap, Lapatinib/capecitabine; WBRT, whole brain radiation therapy; NVB, navelbine; EVE, everolimus; CBP, Carboplatin. (B) Somatic mutations of ctDNA detected by 425 cancer-related genes sequencing. AF, allele frequency.

Figure 2. Characteristics of PIK3R1 mutations from cBioPortal.

The data is analyzed by www.cbioportal.org in all cancer types (A, B) and the breast cancer cohorts (D, E). (C) Malignant tumor patients with PIK3R1 ${ }^{\text {E541_Y542del }}$ are summarized in the table. Location 
of PIK3R1 $1^{\text {E541_Y542del }}$ is indicated in Figure 2B (red arrow).

Figure 1

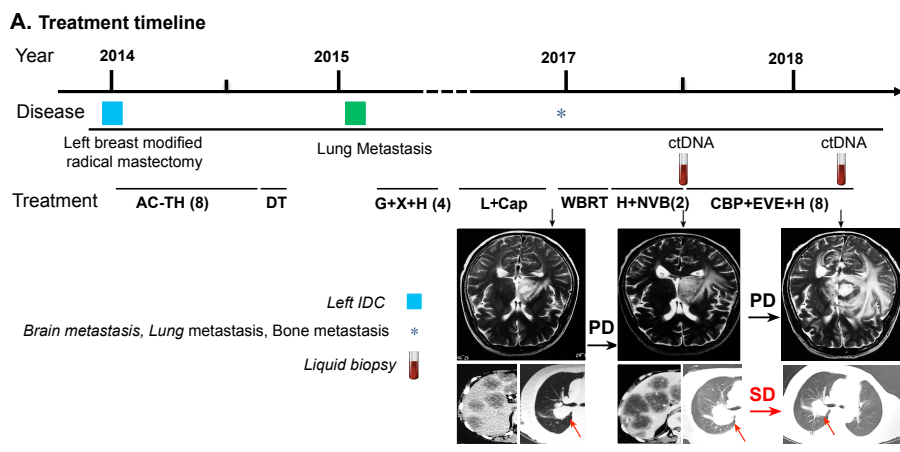

B. Mutational profiling of this breast cancer case.

\begin{tabular}{c|cc|c|c|}
\hline \multicolumn{1}{|c|}{ Gene } & PMT (AF) & & $1^{\text {st }}(2017$ June) & $2^{\text {st }}$ (2018 Jan) \\
\hline \hline PIK3R1 p.EY451delinsD(c.1352_1354delAAT) & $13.3 \%$ & \multicolumn{2}{|c|}{ ctDNA (AF) } \\
\hline TP53 p.V173M (c.G517A) & $37.60 \%$ & $21.8 \%$ & $19.6 \%$ \\
\hline ERBB2 (Copy-Number Variation) & 8.8 & $46.20 \%$ & $49.30 \%$ \\
\hline
\end{tabular}


Figure 2

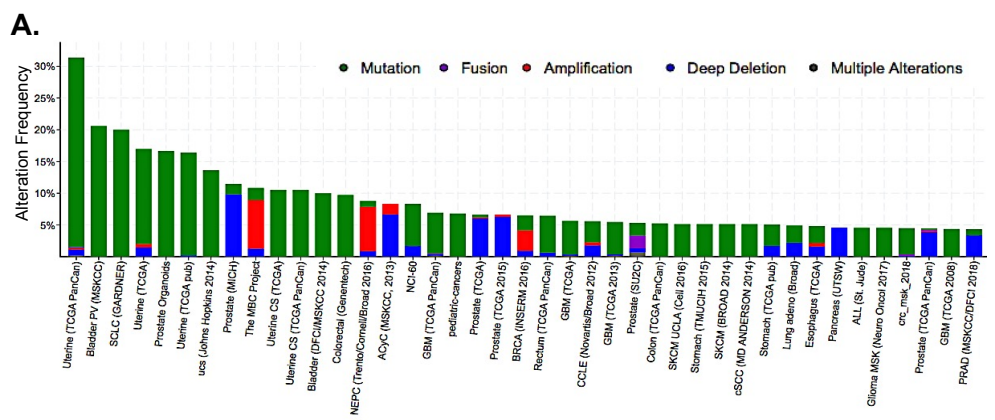

B. PIK3R1

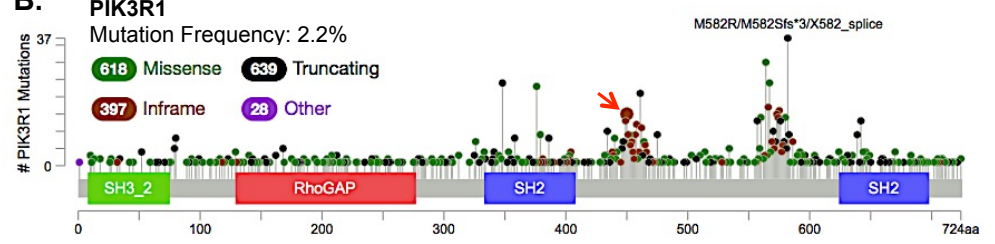

C.

\begin{tabular}{|c|c|c|c|c|}
\hline Case Number & Cancer Type & Protein Change & Allele Freq & Mutation in sample \\
\hline TCGA-CS-5394-01 & Anaplastic Astrocytoma & E451_Y452del & 0.44 & 20 \\
\hline TCGA-AG-3726-01 & Rectal Adenocarcinoma & E451_Y452del & 0.49 & 143 \\
\hline TCGA-A5-A0GQ-01 & Uterine Endometrioid Carcinoma & E451_Y452del & 0.14 & 57 \\
\hline TCGA-B5-A11E-01 & Uterine Endometrioid Carcinoma & E451_Y452del & 0.26 & 9047 \\
\hline TCGA-CS-5394-01 & Diffuse Glioma & E451_Y452del & 0.43 & 19 \\
\hline P-0011284-T01-IM5 & Esophageal Adenocarcinoma & E451_Y452del & 0.14 & 7 \\
\hline P-0001680-T02-IM3 & Lung Squamous Cell Carcinoma & E451_Y452del & - & 11 \\
\hline P-0001680-T01-IM3 & Lung Squamous Cell Carcinoma & E451_Y452del & - & 8 \\
\hline P-0002022-T01-IM3 & Glioblastoma Multiforme & E451_Y452del & - & 6 \\
\hline P-0003600-T01-IM5 & Glioblastoma Multiforme & E451_Y452del & - & 3 \\
\hline SJHGG024-R & B-Lymphoblastic Leukemia & E451_Y452del & - & 25 \\
\hline
\end{tabular}

D.

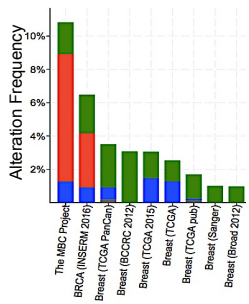

E. PIK3R1 Mutation Frequency: 1.8\%

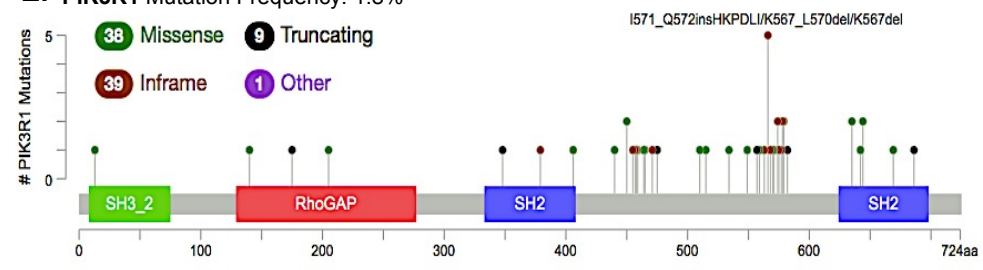

